\title{
Evaluation of sixteen years of INGV seismic bulletins
}

\author{
Rosalba Di Maro \\ Istituto Nazionale di Geofisica e Vulcanologia, Roma, Italy
}

\begin{abstract}
This work analyzes earthquake parameters published in the Seismic Bulletin of the Istituto Nazionale di Geofisica e Vulcanologia (INGV). The analysis and interpretation of the digital signals, done by specialist employees daily, produce most of the seismological information that comprises INGV's earthquake bulletins. After a brief introduction on the criteria we use to obtain seismic parameters, this paper will review the processing procedures employed over a period of sixteen years from 1988 to September 2003. This study also addresses the issue of the comparison between magnitude calculated on signal duration $\left(M_{d}\right)$ and on amplitude $\left(M_{L}\right)$ and the lack of a correct calibration between them. A completeness analysis of the whole bulletin performed using both the Stepp and Habermann techniques shows the importance of considering changes in the seismicity rate and in the geometry of the seismological network. To conclude this excursus, we calculated the errors of hypocentral locations and the detection capacity of individual seismic stations. This final step stresses the increasing improvement of the INGV seismic network over the past 16 years.
\end{abstract}

Key words Seismic Bulletin - magnitude - completeness analysis - errors of hypocentral locations detection capacity

\section{Introduction}

During the first five years of the nineties, the Istituto Nazionale di Geofisica (nowadays INGV) was involved in a European project named «EUROBULL». The aim of the EUROBULL group was to compile a high quality European bulletin to be used both as a reference, to evaluate bulletins produced by international networks, and for scientific purposes.

Italy, France and Sweden collected data from about fifteen countries and, starting from their own bulletin procedures, processed the data independently from each other.

Mailing address: Dr. Rosalba Di Maro, Istituto Nazionale di Geofisica e Vulcanologia, Via di Vigna Murata 605, 00143, Roma, Italy; e-mail: rosalba.dimaro@ingv.it
I was directly involved in the software implementation and Giuseppe Smriglio, a very helpful person who was the manager of the Data Centre, let me modify the Italian bulletin procedures adapting them to the European purposes.

Moreover, the EUROBULL group produced a series of control routines to test the quality of the new bulletin, so we had the opportunity to apply the same test to the Italian bulletin.

This work, that analyzes sixteen years of IN$\mathrm{GV}$ bulletins, recalls the efforts to improve the network and the quality of the data for which Giuseppe was one of the principal promoters.

\section{Database}

Bulletins produced by INGV between 1988 and September 2003, are the database of the present work. Figure 1 shows the area analyzed and the INGV National Centralised Seismic Network (RSNC) configuration used in this paper.

Figure 2 illustrates the evolution of the RSNC during the analyzed period. Colors of the stacked 

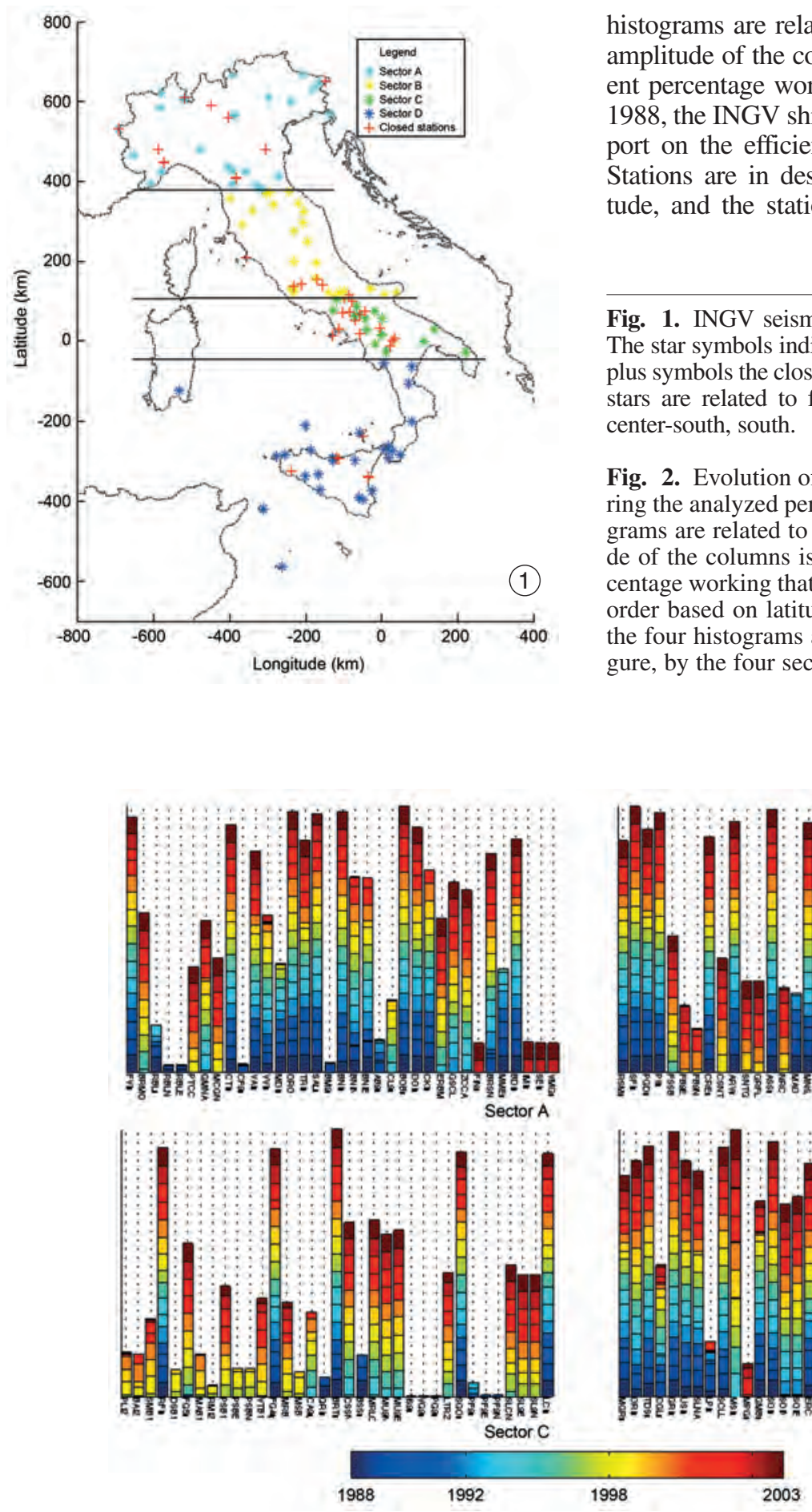

histograms are related to the years, the different amplitude of the columns is related to the different percentage working that year (since January 1988, the INGV shift workers draw up a daily report on the efficiency of each seismic station). Stations are in descending order based on latitude, and the stations reported in the four his-

Fig. 1. INGV seismographic network configuration. The star symbols indicate the station locations, the red plus symbols the closed stations. The four colors of the stars are related to four sectors: north, center-north, center-south, south.

Fig. 2. Evolution of the seismographic network during the analyzed period. Colors of the stacked histograms are related to the years, the different amplitude of the columns is associated to the different percentage working that year. Stations are in descending order based on latitude, and the stations reported in the four histograms are indicated, in the previous figure, by the four sectors respectively.
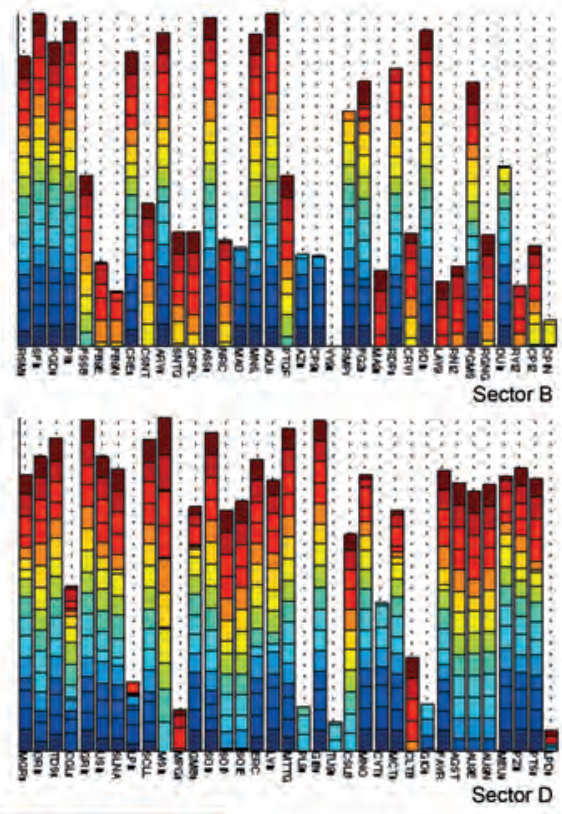
tograms are indicated, in the previous figure, by the four sectors respectively. It is easy to identify from the color bars closed stations and new ones, most of which were installed in Central Italy.

\section{Local magnitude and duration magnitude}

Before processing the database, a brief mention of the magnitude we used in our determinations is useful. For local events we apply the magnitude-duration relation (Console et al., 1988)

$$
M_{d_{i}}=a * \log _{10}\left(\tau_{i}+c \Delta_{i}\right)-b
$$

where $a=2.0, b=0.870$ and $c=0.082 . \tau_{i}$ is the $i$ th seismic signal duration and $\Delta_{i}$ is the linear

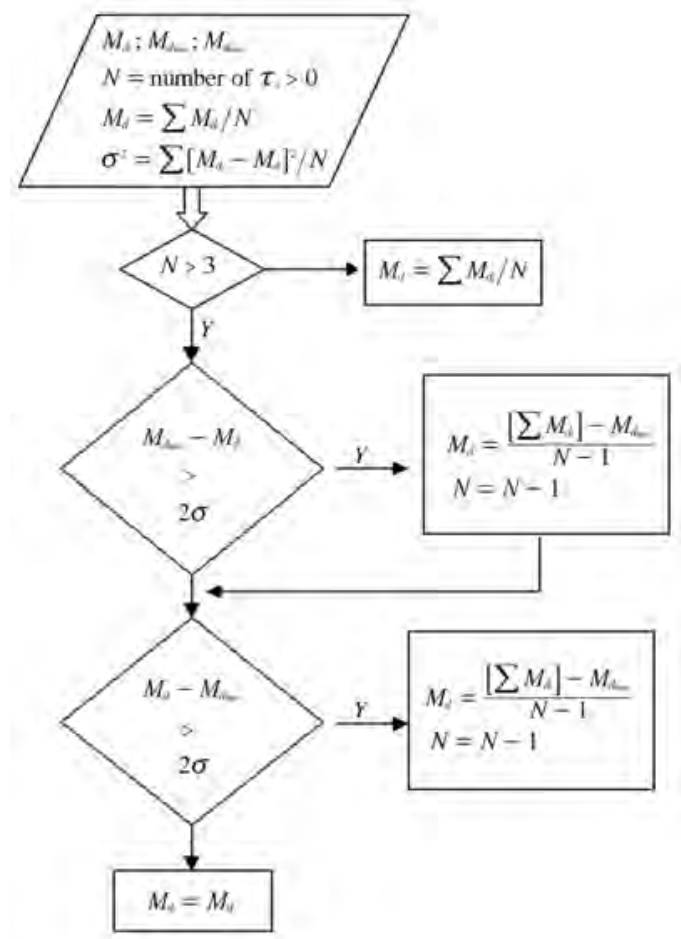

Fig. 3. Flowchart of the algorithm that attributes the $M_{d}$ value to a regional event. This quantity is calculated as the arithmetic average eventually removing the maximum and (/or) the minimum, if they are «very distant» from the average. distance of the $i$-th station compared to the epicenter determination. The (3.1) is derived from the observation that the absolute time $\left(\tau_{i}+c \Delta_{i}\right)$ is constant, in other words independent from the station. Most of the time, the automatic acquisition system calculates $\tau_{i}$ determining the end of the signal when its amplitude comes back to the noise level. When the signal duration exceeds 8-min (that is the maximum acquisition time) the operator evaluates the duration directly from the analog acquisition that has a continuous registration on the paper.

The flowchart in fig. 3 represents the algorithm that attributes $M_{d}$ value to a regional event. This quantity is calculated as arithmetic average among all the values. The maximum and (/or) the minimum are eventually removed if they are «very distant» from the average.

The algorithm that attributes a local magnitude to an event (fig. 3), is the same as the one for $M_{d}$. In this case we eliminate values less than 1.5. The Richter formula is used for stations whose distances, from the epicenter, are less than $600 \mathrm{~km}$

$$
M_{l_{i}}=\log _{10} \frac{\left(A_{N S}+A_{E W}\right)_{i}}{2}+f(\Delta) .
$$

The distance function $f(\Delta)$ is the same published by Richter in 1935 (Richter, 1935). On the other hand, the deduced attenuation function for Italy closely corresponds to Richter's one, as described by Gasperini (2002).

At the present time the RSNC is mostly composed of short period vertical seismometers (S-13 GEOTECH). For this reason, in eq. (3.2), we use the maximum amplitude picked up automatically on the vertical seismic signal and corrected for the instrumental response, as described below.

If $A^{s p}$ is the short period maximum amplitude and $H^{s p}$ the transfer function of the whole instrumental chain, we can obtain the Wood Anderson amplitude (nm) by

$$
A_{i}=A_{i}^{s p} \frac{H^{W A}}{H_{i}^{s p}}
$$

where $H^{W A}$ is the Wood Anderson transfer function.

For regional events $(600-1600 \mathrm{~km})$ we apply a modified Nuttli formula (Nuttli, 1973) 


$$
\left(M_{b L C}\right)_{i}=-4.37+2.34 * \log _{10}\left(\Delta_{i}\right)+\log _{10}\left(\frac{A_{i}}{T_{i}}\right) .
$$

The original formula was modified by means of regression with Italian data (1988-1993).

\section{Data processing}

\subsection{Discrepancy between the duration and local magnitude}

In this paper we processed sixteen years of data: from January 1988 to September 2003 (inclusive). The INGV bulletin reports, for that period, 42154 locatable events («locatable» means that the event has been located using at least six phases). Among them, 23595 events have both local and duration magnitudes and 9594 have at least five $M_{d}$ values. Figure 4 illustrates the annual trend of these quantities.

$M_{L}$ and $M_{d}$ are very different from each other most of the time. Figure 5a,b illustrates this discrepancy: the first displays the frequency of the events for each class of magnitude $M_{d}$ and $M_{L}$. It is easy to notice that the $M_{L}$ histogram is shifted more to the left compared to the $M_{d}$ ones. The dif- ferent shape of the two histograms, provided by the same event data-set, shows that the Italian seismicity in the last sixteen years is concentrated between 2.5 and 3.2 considering $M_{d}$, but under 2.5 if we consider $M_{L}$. Figure $5 \mathrm{~b}$ illustrates that $M_{d}$ and $M_{L}$ are not univocally determined. Also the most probable value seems to be the medium one, considering $M_{d}$ in lack of $M_{L}$ creates a non homogeneous data set.

This ambiguity is due to the method of deriving $M_{d}$. The magnitude-duration relation (3.1)

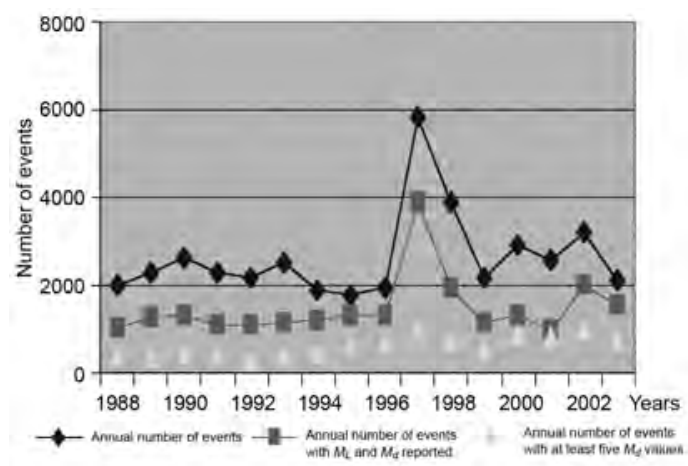

Fig. 4. Annual trend of Italian seismicity.

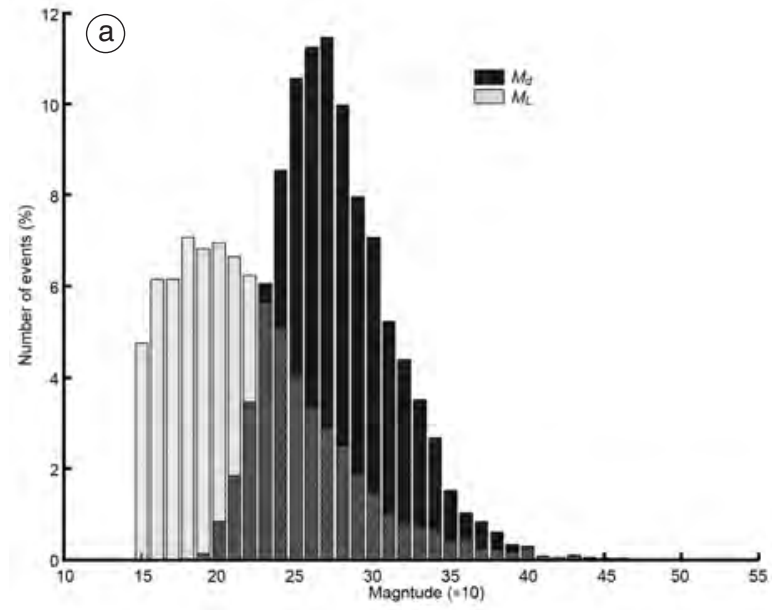

(b)

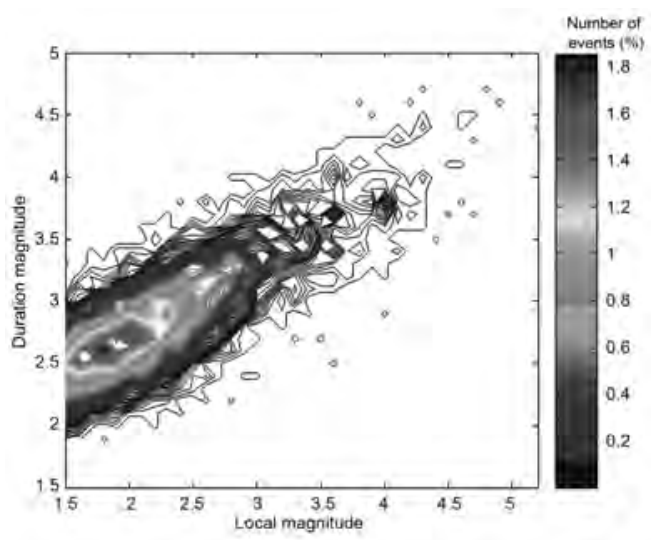

Fig. 5a,b. a) Discrepancy between the frequency of the events for class of magnitude $M_{d}$ and $M_{L}$. b) Three-dimensional vision of the correspondences between $M_{d}$ and $M_{L}$. 


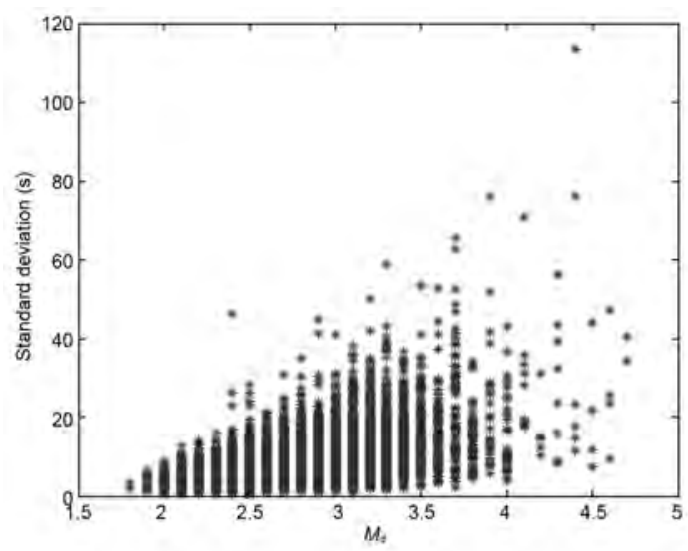

Fig. 6. Standard deviations of the absolute time $\left(\tau_{s}\right)$ versus $M_{d}$. The standard deviation values indicate that $\tau_{s}$ is not constant.

has been obtained analyzing 15 months of data that are probably not enough for a correct determination of parameters. Another problem is related to the new formulation given by (3.1) of the absolute time.

To validate the hypothesis

$$
\tau_{s}=\frac{\Delta_{s}}{v}=\tau_{i}+\left(T_{i}-T_{0}\right)=\mathrm{const}
$$

where $\tau_{s}$ is the absolute duration; $\Delta_{s}$ is the total distance covered by waveforms that travel with velocity $v ; \tau_{i}$ is the $i$-station signal duration; $T_{i}$ is the $i$-station arrival time; $T_{0}$ is the origin time. We calculated $\tau_{s}$ and the related standard deviations for the whole data set. Figure 6 illustrates this trend, the biggest magnitude values, that have the biggest number of recordings, are associated to high values of standard deviations. This means that the absolute duration is not independent from the station and (4.1) is not validated.

\subsection{Data set completeness}

To determine the completeness of our data set we apply the Stepp method (Stepp, 1972).

This model assumes that:

- Earthquake distribution can be modeled by a Poisson distribution whose standard deviation is

$$
\sigma_{\lambda}=\sqrt{\frac{\lambda}{T}}
$$

where $\lambda$ is the average of the occurrences in the period $T$.

- The seismic activity for the whole observed period is stationary i.e. $\lambda=$ cost.

Let's go back to our data set and consider first the local magnitude. Observing the seismicity year by year, four steps of local magnitude and duration magnitude have been selected

$$
\begin{array}{cc}
M_{d} \leq 2.4 & M_{L} \leq 1.8 \\
2.5 \leq M_{d} \leq 2.8 & 1.9 \leq M_{L} \leq 2.4 \\
2.9 \leq M_{d} \leq 3.4 & 2.5 \leq M_{L} \leq 3.0 \\
M_{d} \geq 3.5 & M_{L} \geq 3.1 .
\end{array}
$$

Figures $7 \mathrm{a}$ and $8 \mathrm{a}$ show the cumulated number of events during the 16-year period, whereas figs. $7 \mathrm{~b}$ and $8 \mathrm{~b}$ show, in bi-logarithmic scale, $s$ versus $T$ (starting from 2003).

According to Stepp's theory, when the mean rate of occurrences is constant, the related period of time is 'complete' and (4.2) has the linear trend shown in the figs. $7 \mathrm{~b}$ and $8 \mathrm{~b}$, as reference, in green. The four steps of magnitude $M_{d}$ have the same «critical» time $T_{c}$ under which the data set is considered incomplete. The four steps of magnitude $M_{L}$ have two «critical» times, one, in common with $M_{d}$ corresponding to 1997 , and the other for $M_{L} \geq 3$.1, corresponding to 1999 .

The explanation for this «critical» year could derive from fig. 2: numbers of stations were added, during 1997, in the middle-south part of Italy. Consequently, an apparent low seismicity has grown during the last period. The increment in the station density makes the data set for the period before 1997 «incomplete». Moreover we can make another comment: Stepp's approach to the data-set completeness has the «Poissonianity» of the event distribution underlying the hypothesis. Of course clusters of events as well as aftershocks or foreshocks are not independent, so their spatial, temporary distributions are not «Poissonian».

In addition we should mention the "Colfiorito» sequence that contributed with more than 5000 events during the 1997 and 1998.

Of course it is possible to make the database «Poissonian» removing the clusters, but this is not the purpose of this paper. 


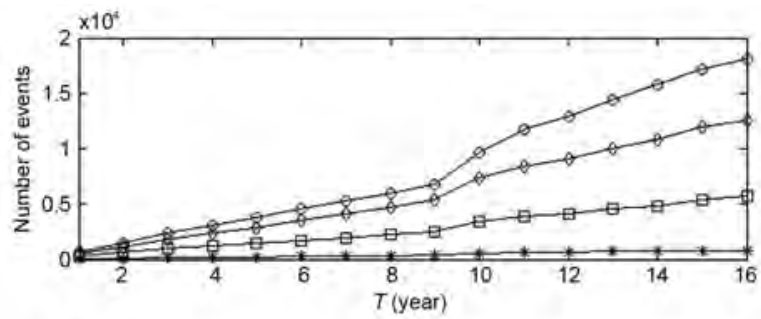

(a)

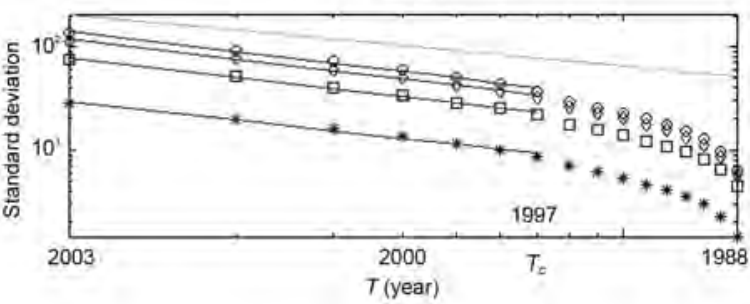

Legend

- $M_{0} \leq 2.4$

- $2.5 \leq M_{d} \leq 2,8$

a $2.9 \leq M_{d} \leq 3.4$

- $M_{c} \geq 3,5$

- Theoretical trend

- Linear regr.

Fig. 7a,b. a) Cumulated number of events during the 16-year period for four steps of duration magnitude. b) Standard deviation, versus $T$ (starting from 2003) in bi-logarithmic scale for four steps of duration magnitude.

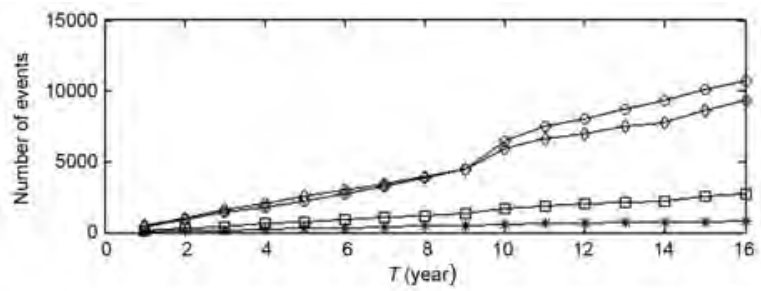

(a)

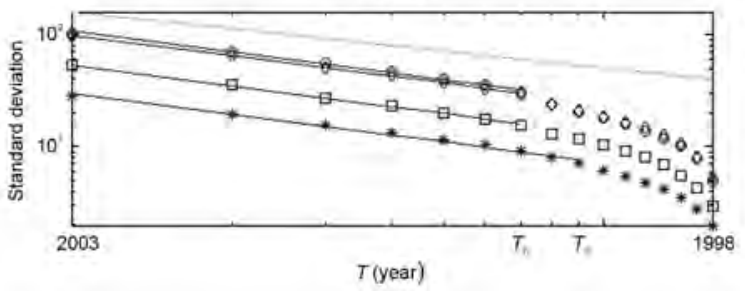

Legend

- $M_{d} \leq 1.8$

- $1.9 \leq M_{d} \leq 2.4$

b $2.5 \leq M_{d} \leq 3.0$

- $M_{d} \geq 3.1$

- Theoretical trend

- Linear regr.

Fig. 8a,b. a) Cumulated number of events during the 16-year period for four steps of local magnitude. b) Standard deviation, versus $T$ (starting from 2003) in bi-logarithmic scale for four step of local magnitude.

Habermann (1983) proposed another definition for the minimum magnitude of completeness.

Beginning from the Gutenberg-Richter (1944) relationship

$$
\log _{10} N=a-b M .
$$

The determination of the minimum magnitude of completeness $M_{C}$ of a data set is obtained plotting (4.3) where $N$ is the cumulative number of events, $M$ the magnitude and $a$ and $b$ are constants. Figure 9a,b shows the frequency-magnitude distribution of Italian earthquakes during these sixteen years. The straight line represents the best fit of the observations in the area of «complete» recording indicated by $M_{C}$. We can individuate this 

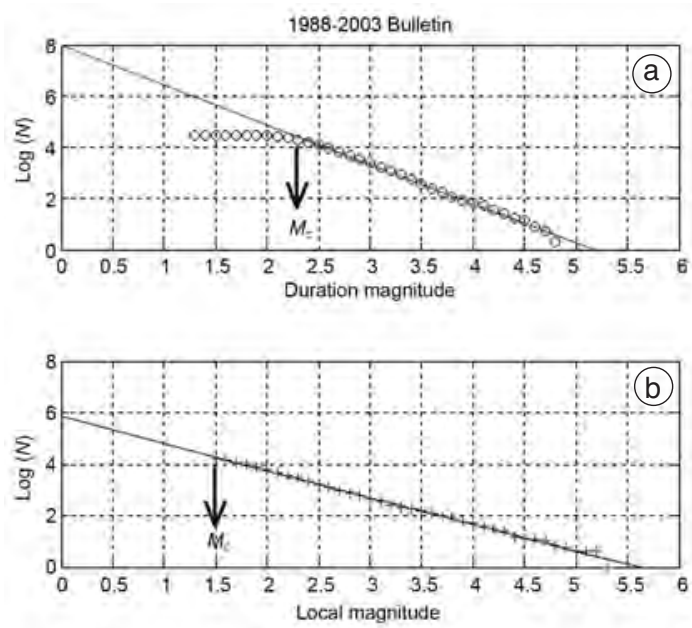

Fig. 9a,b. a) Minimum magnitude of completeness for $M_{d}$ in accordance with Habermann (1983). b) Minimum magnitude of completeness for $M_{L}$ in accordance with Habermann (1983). values that for $M_{L}$ coincides with the minimum value 1.5 and for $M_{d}$ is $2.3 \pm 0.1$.

Performing the regression (for both relations the correlation coefficient is approximately 1 ) we can write (4.3) as follows:

$$
\begin{aligned}
& \log _{10} N=-1.54 M_{d}+8.0 \\
& \log _{10} N=-1.03 M_{L}+5.8
\end{aligned}
$$

\subsection{Hypocentral locations}

As mentioned before, in these sixteen years of bulletins, our qualified technicians processed more than 40000 events, merging data from several seismic networks to obtain a high quality product.

The hypocentral determinations are performed, using routines belonging to the Hypo71 family.

\begin{tabular}{|c|c|c|c|c|c|c|c|c|c|c|c|c|c|c|c|c|c|c|c|}
\hline Jan & 1 & $\underset{R}{C a t}$ & $\begin{array}{r}\text { Nst } \\
6\end{array}$ & $\begin{array}{r}\text { Ndi } \\
10\end{array}$ & $\begin{array}{r}\text { Ndf } \\
10\end{array}$ & $\begin{array}{l}\text { Md } \\
28\end{array}$ & \multicolumn{2}{|c|}{$\underset{4}{n}$} & $\begin{array}{rr}\frac{1}{2} & \mathrm{nMl} \\
2 & 3 \\
3>\end{array}$ & ADR & IATIC & $\begin{array}{r}42.642 \\
3.684 \\
100 \text { CEN }\end{array}$ & $\begin{array}{l}2 \text { Lon } \\
4 \text { TTRALE }\end{array}$ & $\begin{array}{c}15,696 \\
5,317 \\
68\end{array}$ & Dep & $\begin{array}{c}10.000 \text { To } 04 \\
\text { Fixed }\end{array}$ & $\begin{array}{l}4: 01: 27.52 \\
0,760\end{array}$ & $\begin{array}{l}\text { Gap } \\
294\end{array}$ & $\begin{array}{l}\text { Res } \\
0.5\end{array}$ \\
\hline Sta & $\mathbf{P}$ & Phasel & v & \multicolumn{2}{|c|}{ Arrivall } & Res & \multicolumn{2}{|r|}{ Del } & $\mathrm{Azm}$ & Dux & $\mathrm{Hd}$ & Anp & Per & H1 Phase2 & 2. V & Arrival2 & Res & & \\
\hline $\begin{array}{l}\text { FG2 } \\
\text { RGVG } \\
\text { RHI2 } \\
\text { SDI } \\
\text { VTB1 } \\
\text { PTQR }\end{array}$ & & $\begin{array}{ll}E Z & P G \\
E Z & P G \\
E Z & P N \\
E Z & P N \\
E Z & P N \\
E Z & P H\end{array}$ & $\begin{array}{ll}0 & 8 \\
2 & 0 \\
1 & 6 \\
1 & 2 \\
1 & 2 \\
1 & 8\end{array}$ & $\begin{array}{ll}4 & 01 \\
4 & 01 \\
4 & 01 \\
4 & 01 \\
4 & 01 \\
4 & 01\end{array}$ & $\begin{array}{l}4496 \\
4656 \\
5426 \\
5605 \\
5689 \\
5840\end{array}$ & $\begin{array}{r}-0.82 \\
-0.05 \\
0.38 \\
-0.57 \\
0.53 \\
0.05\end{array}$ & & $\begin{array}{l}102 \\
107 \\
164 \\
186 \\
194 \\
200\end{array}$ & $\begin{array}{l}205 \\
184 \\
230 \\
236 \\
208 \\
250\end{array}$ & $\begin{array}{l}54 \\
64 \\
57 \\
67 \\
58\end{array}$ & $\begin{array}{l}28 \\
28 \\
28 \\
29 \\
28\end{array}$ & $\begin{array}{r}316 \\
41 \\
31 \\
34 \\
23\end{array}$ & $\begin{array}{l}0.28 \\
0.40 \\
0.54 \\
0.46 \\
0.64\end{array}$ & $\begin{array}{lll}29 & \mathrm{EZ} & \mathrm{SG} \\
21 & \mathrm{EZ} & \mathrm{SG} \\
22 & \mathrm{EZ} & \mathrm{SN} \\
24 & \mathrm{EZ} & \mathrm{SN} \\
21 & & \end{array}$ & $\begin{array}{l}0.5 \\
0.3 \\
0.6 \\
0.1\end{array}$ & $\begin{array}{lll}4 & 01 & 5860 \\
4 & 02 & 0144 \\
4 & 02 & 1268 \\
4 & 02 & 1926\end{array}$ & $\begin{array}{r}-0.55 \\
0.85 \\
-0.49 \\
1.33\end{array}$ & & \\
\hline Jan & 1 & $\underset{I}{\text { Cat }}$ & $\begin{array}{r}\text { Nst } \\
6\end{array}$ & $\begin{array}{r}\text { Ndi } \\
10\end{array}$ & $\begin{array}{r}\text { Ndt } \\
10\end{array}$ & $\frac{\mathrm{Md}}{24}$ & nMd & $\frac{\mathrm{M} 1}{25}$ & $\frac{1}{5} \underset{3}{3} \frac{1}{2}$ & $\frac{1}{4}$ & RENO & $\begin{array}{r}38.374 \\
1.692 \\
\text { MERID }\end{array}$ & $\frac{1}{4}+\frac{\operatorname{Lon}}{4-}$ & $\begin{array}{c}13,648 \\
1.568 \\
<6\end{array}$ & $\begin{array}{l}\text { Dep } \\
+/=\end{array}$ & $\begin{array}{ll}5.000 & \text { To } 13 \\
\text { Fived } & +/-\end{array}$ & $\begin{array}{l}3: 43-30,71 \\
0,266\end{array}$ & $\begin{array}{l}\text { Gap } \\
145\end{array}$ & $\begin{array}{l}\text { Ras } \\
0.6\end{array}$ \\
\hline Sta & P & Phase 1 & $v$ & Arriv & Val1 & Res & & Det & $A z \mathrm{a}$ & Dux & Md & Amp & Per & M1 Phase2 & $2 v$ & Arrival2 & Res & & \\
\hline $\begin{array}{l}\text { MPG } \\
\text { GIB } \\
\text { USI } \\
\text { CSLB } \\
\text { CITB } \\
\text { SOI }\end{array}$ & & $\begin{array}{l}E Z P G \\
E Z P G \\
E Z P G \\
E Z P G \\
E Z P G \\
E Z P N\end{array}$ & $\begin{array}{ll}1 & 7 \\
1 & 7 \\
1 & 8 \\
1 & 1 \\
0 & 6 \\
0 & 5 \\
0 & 5\end{array}$ & $\begin{array}{ll}13 & 43 \\
13 & 43 \\
13 & 43 \\
13 & 43 \\
13 & 43 \\
13 & 44\end{array}$ & $\begin{array}{l}3720 \\
4085 \\
4113 \\
4159 \\
4952 \\
0464\end{array}$ & $\begin{array}{r}-0.50 \\
-0.52 \\
-0.45 \\
-0.82 \\
1.10 \\
0.83\end{array}$ & & $\begin{array}{r}34 \\
54 \\
55 \\
50 \\
96 \\
212\end{array}$ & $\begin{array}{r}227 \\
141 \\
312 \\
143 \\
203 \\
98\end{array}$ & $\begin{array}{l}39 \\
45 \\
38 \\
49\end{array}$ & $\begin{array}{l}23 \\
25 \\
23 \\
25\end{array}$ & $\begin{array}{r}225 \\
883 \\
465 \\
16\end{array}$ & $\begin{array}{l}0.30 \\
0.30 \\
0.44 \\
0.30\end{array}$ & $\begin{array}{lll}21 & \mathrm{EZ} & 56 \\
& \mathrm{EZ} & 56 \\
30 & \mathrm{EZ} & 56 \\
28 & \mathrm{EZ} & 56 \\
22 & \end{array}$ & $\begin{array}{l}0.9 \\
0.4 \\
0.7 \\
0.7\end{array}$ & $\begin{array}{lll}13 & 43 & 4293 \\
13 & 43 & 5003 \\
13 & 43 & 4994 \\
13 & 43 & 5047\end{array}$ & $\begin{array}{r}0.12 \\
0.86 \\
0.41 \\
-0.51\end{array}$ & & \\
\hline Jan & 1 & Cat & $\begin{array}{r}\text { Nst } \\
6\end{array}$ & $\frac{N d i}{10}$ & $\underset{10}{\mathrm{Ndf}}$ & $\mathrm{Md}_{26}$ & nifd & $\begin{array}{l}\text { M1 } \\
17\end{array}$ & $\frac{1}{7} \underset{3}{3}$ & $\begin{array}{l}\mathrm{I} \\
\text { FUC }\end{array}$ & & $\begin{array}{r}41.896 \\
1.468\end{array}$ & $\begin{array}{l}\text { Lon } \\
3+{ }^{2}-\end{array}$ & $\begin{array}{c}13.691 \\
1.512 \\
<5\end{array}$ & $\begin{array}{l}\text { Dep } \\
+\rightarrow-\end{array}$ & $\begin{array}{c}14.436 \text { To } 20 \\
1.838 \text { t }=\end{array}$ & $\begin{array}{l}0.23: 40.25 \\
0.137\end{array}$ & $\begin{array}{l}\text { Gap } \\
144\end{array}$ & $\begin{array}{l}\text { Ras } \\
0,1\end{array}$ \\
\hline Sta & $\mathrm{P}$ & Phase1 & $v$ & Arriv & vali & Res & & Del & Azn & Dur & $\mathrm{Md}$ & Amp & Per & M1 Phase2 & 2 V & Arrival2 & Res & & \\
\hline $\begin{array}{l}\text { VVID } \\
\text { SDI } \\
\text { PTQR } \\
\text { RHI2 } \\
\text { AOD } \\
\text { MNS }\end{array}$ & D & $\begin{array}{ll}E Z & P G \\
I Z & P G \\
E Z & P G \\
E Z & P G \\
E Z & P G \\
E Z & \text { PG }\end{array}$ & $\begin{array}{ll}1 & 5 \\
3 & 5 \\
0 & .4 \\
1 & 4 \\
0 & 8 \\
0 & .4\end{array}$ & $\begin{array}{ll}20 & 23 \\
20 & 23 \\
20 & 23 \\
20 & 23 \\
20 & 23 \\
20 & 23\end{array}$ & $\begin{array}{l}4336 \\
4541 \\
4572 \\
4858 \\
5099 \\
5822\end{array}$ & $\begin{array}{r}0.10 \\
0.05 \\
-0.38 \\
-0.13 \\
0.23 \\
0.27\end{array}$ & & $\begin{array}{r}6 \\
22 \\
27 \\
43 \\
56 \\
99\end{array}$ & $\begin{array}{l}241 \\
154 \\
300 \\
119 \\
334 \\
303\end{array}$ & $\begin{array}{l}50 \\
63 \\
59 \\
52 \\
50 \\
54\end{array}$ & $\begin{array}{l}25 \\
27 \\
27 \\
26 \\
26 \\
27\end{array}$ & $\begin{array}{l}56 \\
28 \\
24\end{array}$ & $\begin{array}{l}0.23 \\
0.20 \\
0.42\end{array}$ & $\begin{array}{rrr} & E Z & 56 \\
& E Z & 56 \\
& E Z & 56 \\
17 & E Z & 56 \\
16 & & \\
18 & & \end{array}$ & $\begin{array}{l}0.7 \\
0.7 \\
0.1 \\
0.4\end{array}$ & $\begin{array}{lll}20 & 23 & 4552 \\
20 & 23 & 4916 \\
20 & 23 & 4997 \\
20 & 23 & 5470\end{array}$ & $\begin{array}{r}0.07 \\
0.07 \\
-0.42 \\
-0.20\end{array}$ & & \\
\hline
\end{tabular}

Fig. 10. The first three events of the 2003 INGV Bulletin. 
Figure 10 shows an extract of the INGV Bulletin, in particular the first three events of 2003.

Most of the events have the location fixed at a certain depth, sometimes the software itself

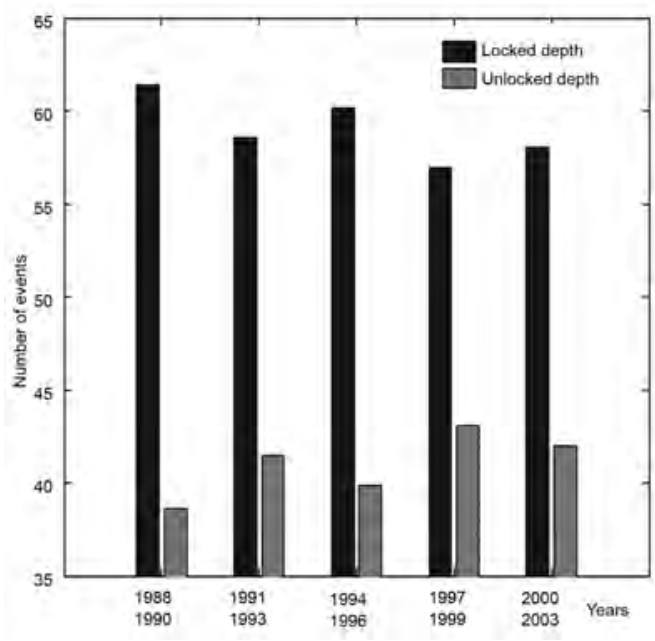

Fig. 11. Percentage of the events whose hypocentral location has fixed (darker) depth. The percentages are calculated over a three year period, as indicated on the abscissa. fixes the depth, and sometimes because the depth value is not consistent the technicians fix them manually. By increasing the number of the stations, the number of events with fixed depth is decreasing as the fig. 11 demonstrates. Figure $12 \mathrm{a}$ reports all the depths selected among events with the product of coordinate errors (Lat error $*$ Long error $*$ depth error) below $1000 \mathrm{~km}^{3}$. The interpolation is made on a grid of $30 \mathrm{~km}$ side. From the figure the very deep location of the events in the southern part of the Tyrrhenian Sea is clear. The depth of most of the other events is less than $40 \mathrm{~km}$, as fig. $12 \mathrm{~b}$ shows.

Regarding the errors on the hypocentral locations, the routine we use gives latitude, longitude and depth errors in kilometres instead of the ellipsoid error. To have an idea on the quality of the locations, let us take a look at the error location distribution. Table I shows (in the second column) the percentage of the events with a certain error indicated in the first column.

Most of these events have a very small error, fig. 13a-d show the distribution of the smallmedium location error (below $1000 \mathrm{~km}^{3}$ ) separated for class of duration magnitude, it is remarkable that greatest errors are related with areas not included in the RSNC and, of course,
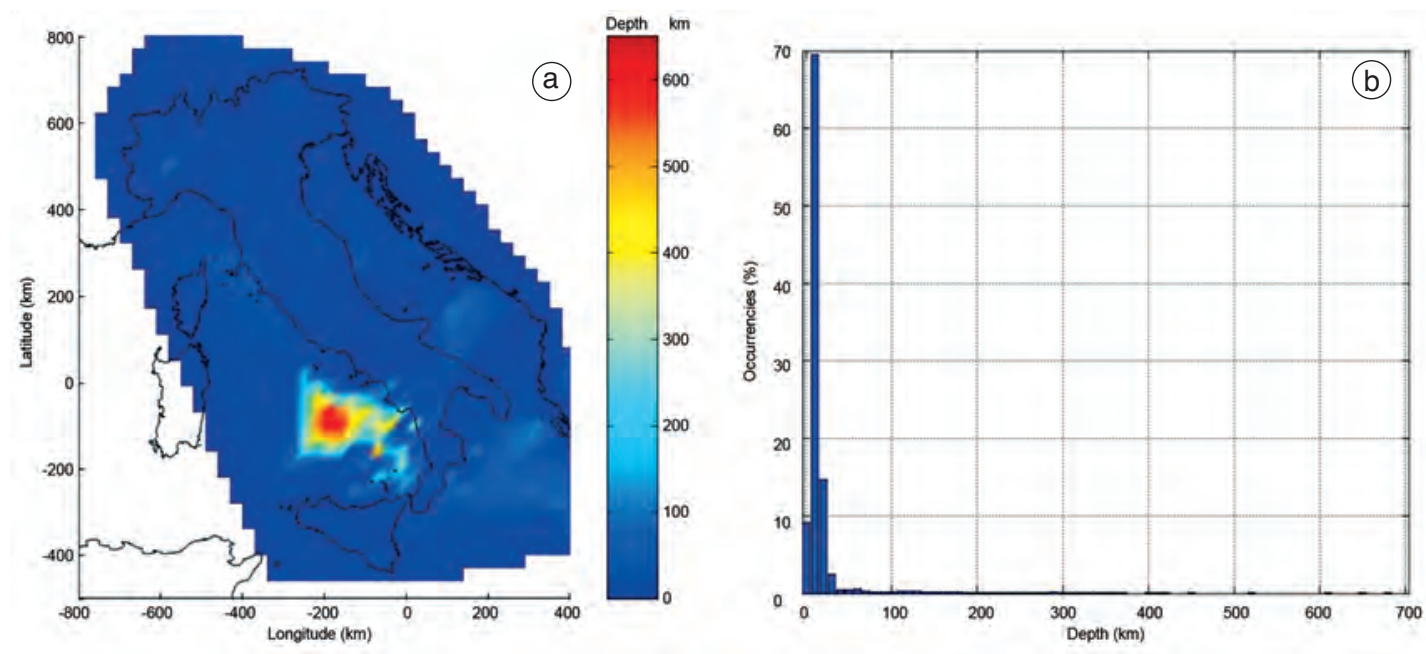

Fig. 12a,b. a) Hypocentral depths map distribution. The events selected have an error on the coordinates (Lat error $*$ Long error $*$ depth error) below $1000 \mathrm{~km}^{3}$. The interpolation is made on a grid of $30 \mathrm{~km}$ per side. b) Hypocentral depths distribution in class of $10 \mathrm{~km}$ each. The events selected have an error on the coordinates (Lat error $*$ Long error $*$ depth error) below $1000 \mathrm{~km}^{3}$. 
Table I. Evaluation of the error associated to hypocentral locations. The percentage only considers the events whose depth is not fixed.

\begin{tabular}{cc}
\hline \hline$V\left(\mathrm{~km}^{3}\right)$ & \\
Lat error $*$ Long error $*$ depth error & Percentage \\
\hline $0 \leq V<100$ & $81.6 \%$ \\
$100 \leq V<1000$ & $15.6 \%$ \\
$V \geq 1000$ & $2.8 \%$ \\
\hline
\end{tabular}

the bigger the event the better the hypocentral location will be.

To complete the «panorama», fig. 13e summarizes everything about the $2.8 \%$ of the largest errors. The radius of the circumferences is increasing with $M_{d}$, the color is darkens with the error values and, in the end, the plus sign indicates the events which occurred in the last three years. We can notice that:
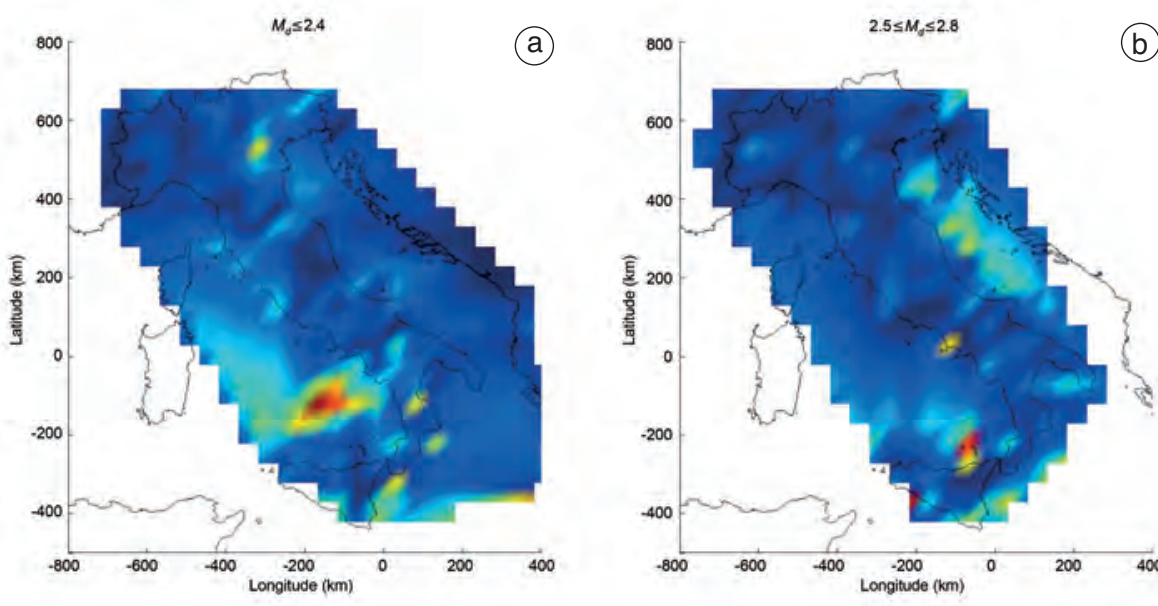

(b)
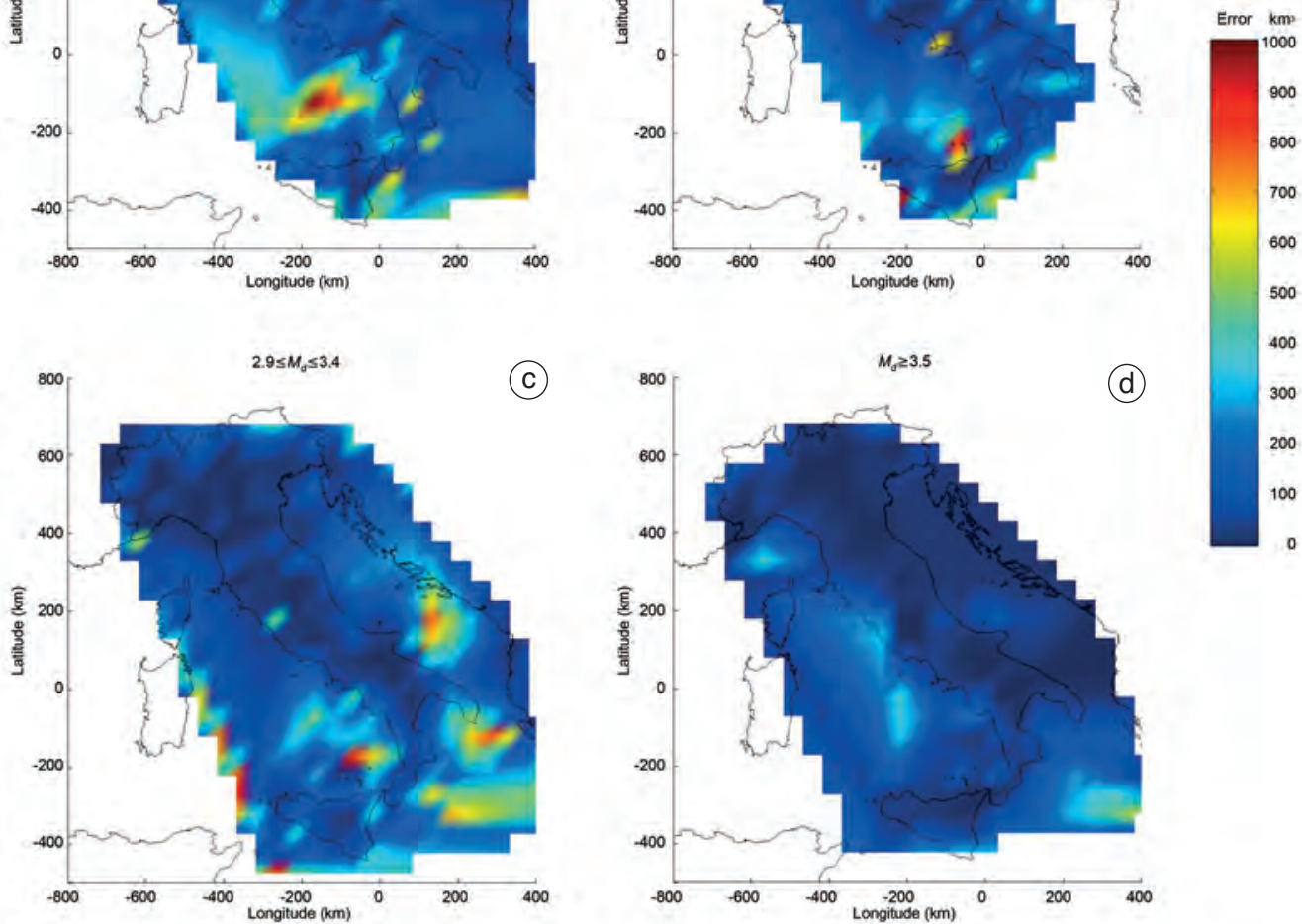

Fig. 13a-d. a) Distribution of the small-medium localization error (below $1000 \mathrm{~km}^{3}$ ) for events with $M_{d} \leq 2.4$. The interpolation is made on a grid of $50 \mathrm{~km}$ per side. b) Distribution of the small-medium localization error (below $1000 \mathrm{~km}^{3}$ ) for events with $2.5 \leq M_{d} \leq 2.8$. The interpolation is made on a grid of $50 \mathrm{~km}$ per side. c) Distribution of the small-medium localization error (below $1000 \mathrm{~km}^{3}$ ) for events with $2.9 \leq M_{d} \leq 3.4$. The interpolation is made on a grid of $50 \mathrm{~km}$ per side. d) Distribution of the small-medium localization error (below $1000 \mathrm{~km}^{3}$ ) for events with $M_{d} \geq 3.5$. The interpolation is made on a grid of $50 \mathrm{~km}$ per side. 


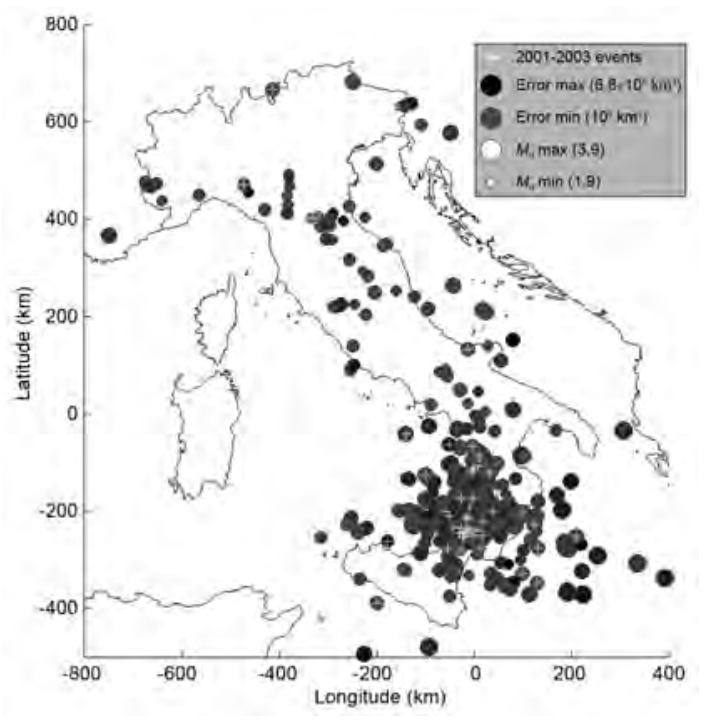

Fig. 13e. Largest errors (above $1000 \mathrm{~km}^{3}$ ) distribution. The radius of the circumferences is related to $M_{d}$ value, the color changes with the error values and, in the end, the plus sign indicates the events which occurred in the last three years (see the legend as reference).

- The hypocentral locations with the biggest error and magnitude are outside the network.

- Most are concentrated in the southern part of the Tyrrhenian Sea where the deepest events occur.

- The increasing number of the stations in the last three years improved the quality of locations, only few of them, with low magnitude, are inside the network.

\subsection{The detection capacity of the seismographic stations}

This last paragraph is dedicated to the performance of the RSNC stations. All the events recorded by each station have been considered, more precisely, all the arrival times that contributed to evaluate the hypocentral location have been considered (not all the phases recorded by the automatic system will contribute to the location process; it depends on the signal/ noise ratio and on the convergence of the location algorithm solution). The events are collected into four steps of duration magnitude, as usual

$$
\begin{gathered}
M_{d} \leq 2.4 \\
2.5 \leq M_{d} \leq 2.8 \\
2.9 \leq M_{d} \leq 3.4 \\
M_{d} \geq 3.5 .
\end{gathered}
$$

The area around each station has been divided into twelve parts $\left(30^{\circ}\right.$ each), each part has been divided into sectors whose dimensions are related to the distance from the center (i.e., the station). Figure 14 explains the criteria.

I selected, to be brief, three stations that, from north to the south are: Trieste (TRI), L'Aquila (AQU) and Sicignano (SGO). The selection was on the location basis and also because these stations are currently working. The complete analysis of all the stations will be the topic of a specific paper.

The results of this partial investigation are reported in fig. 15a-l:

1) SGO has the best coverage for all the steps of magnitude.

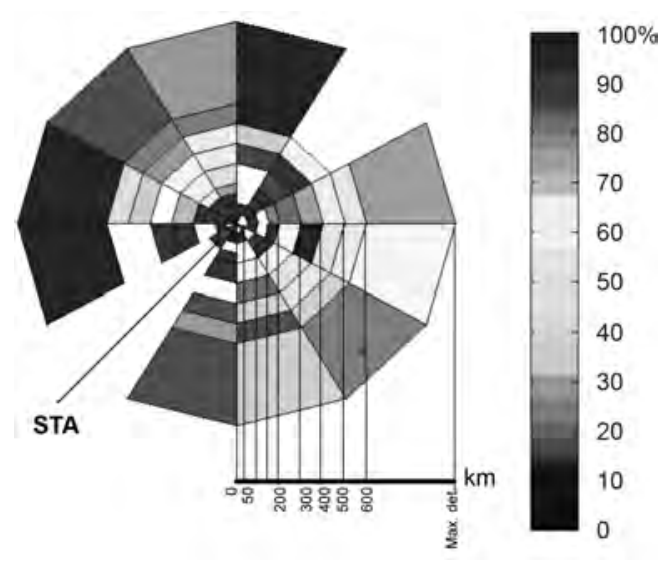

Fig. 14. Criteria to determine the detection capacity of the seismographic stations. The area around each station has been divided in twelve parts ( $30^{\circ}$ each), each part has been divided in to sectors whose dimensions are related to the distance from the station: for the first $200 \mathrm{~km}$ the sectors are $50 \mathrm{~km}$ by $50 \mathrm{~km}$, from $200 \mathrm{~km}$ to $600 \mathrm{~km}$ the distances are $100 \mathrm{~km}$ each. The farthest sector has the distance, from the station, determined by the farthest event detected (marked in the figure by a red or white star). The white sectors, eventually not closed, indicate a lack of events. 

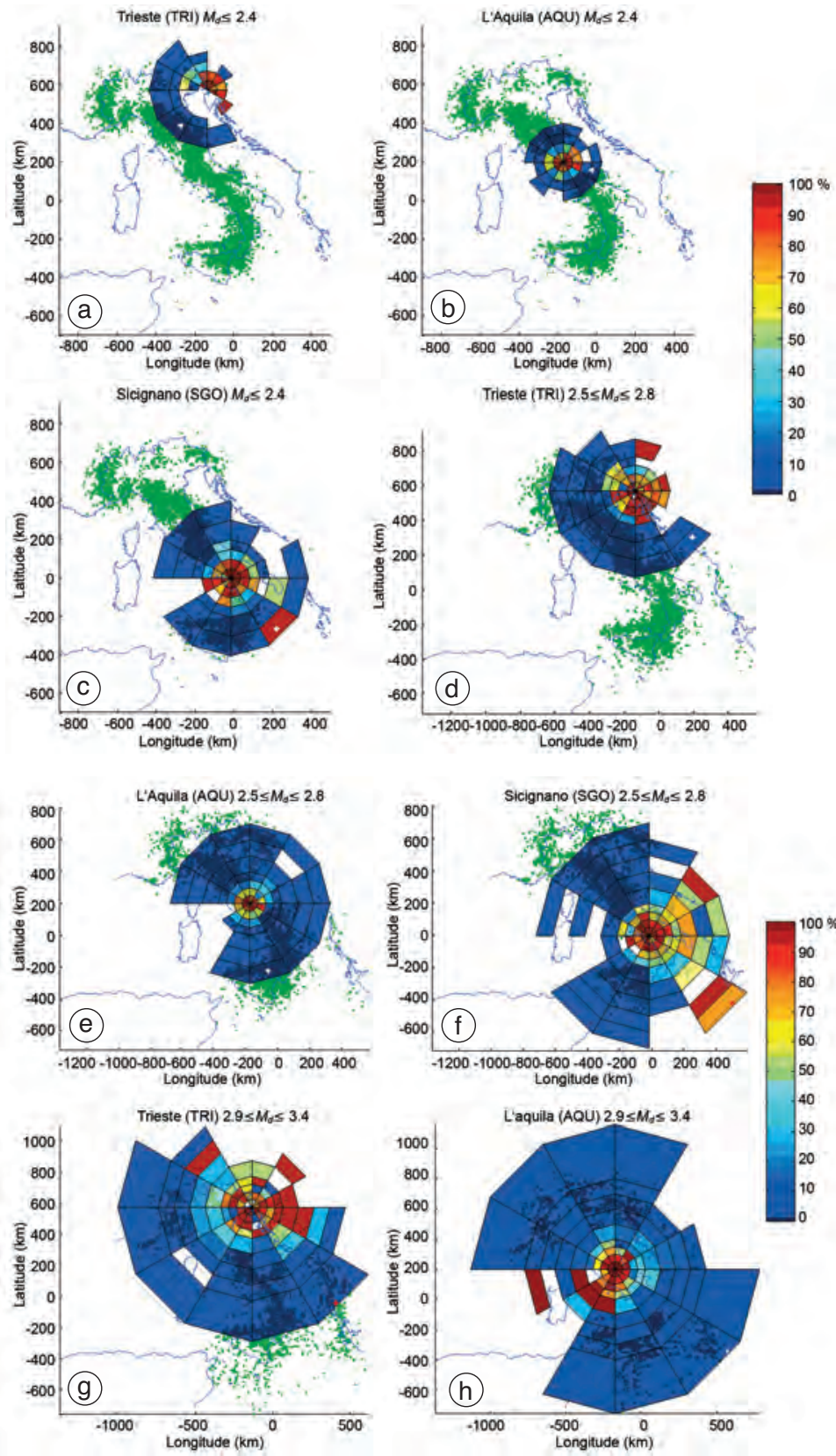

Fig. 15a-h. a) Percentage of events $\left(M_{d} \leq 2.4\right)$ detected by Trieste station. b) Percentage of events $\left(M_{d} \leq 2.4\right)$ detected by L'Aquila station. c) Percentage of events $\left(M_{d} \leq 2.4\right)$ detected by Sicignano station. d) Percentage of events $\left(2.5 \leq M_{d} \leq 2.8\right)$ detected by Trieste station. e) Percentage of events ( $\left.2.5 \leq M_{d} \leq 2.8\right)$ detected by L'Aquila station. f) Percentage of events $\left(2.5 \leq M_{d} \leq 2.8\right)$ detected by Sicignano station. g) Percentage of events $\left(2.9 \leq M_{d} \leq 3.4\right)$ detected by Trieste station. h) Percentage of events $\left(2.9 \leq M_{d} \leq 3.4\right)$ detected by L'Aquila station. The criteria are explained in fig. 14. Green dots are the events recorded by the RSNC network in the range of duration magnitude indicated. 

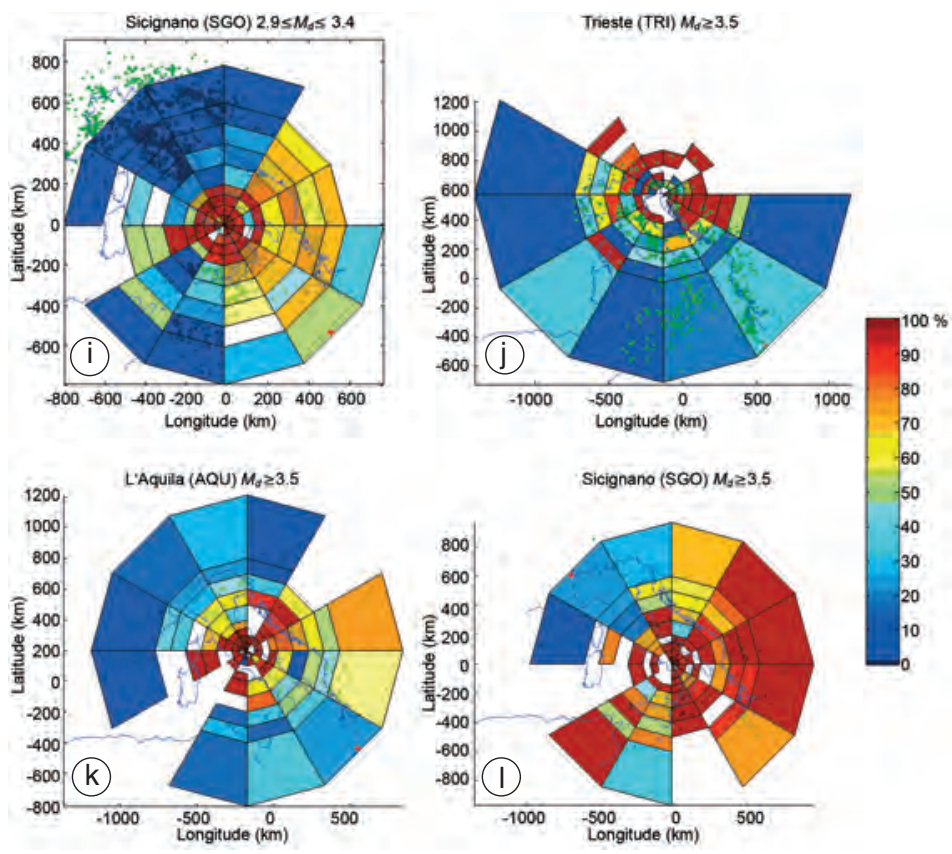

Fig. 15i-l. i) Percentage of events $\left(2.9 \leq M_{d} \leq 3.4\right)$ detected by Sicignano station. j) Percentage of events $\left(M_{d} \geq\right.$ $\geq 3.5)$ detected by Trieste station. k) Percentage of events $\left(M_{d} \geq 3.5\right)$ detected by L'Aquila station. 1) Percentage of events $\left(M_{d} \geq 3.5\right)$ detected by Sicignano station. The criteria are explained in fig. 14. Green dots are the events recorded by the RSNC network in the range of duration magnitude indicated.

2) The farthest event detected is not in a sector with a high percentage of detection.

3) No privileged direction in the detections is visible.

4) A high percentage of detection does not correspond to those areas with a high seismicity.

The «strategy» in the performing of hypocentral location is one possible explanation for the last point. In fact, in case of an abundant number of phases, the technicians exclude those arrival times belonging to far stations in order to obtain a robust solution for the location algorithm. This could be the reason for the second point also.

\section{Conclusions}

Sixteen years ago, the possibility to acquire and process digital seismic signals gave a new impulse and speeded up the process of optimization of the RSNC.
This overview emphasizes the effort to improve the software and the hardware related to the correct detection and characterization of the earthquakes.

Regarding the parameters of the hypocentral location the accuracy is not under discussion. The only weak link in the chain is, as indicated above, the magnitude and the correlation between the local and duration magnitude.

Nowadays the introduction of the new digital seismographic network, with new sensors, three-components and broadband, will allow a more correct definition of the local magnitude, so we could calibrate the duration magnitude having much more data to compare.

\section{REFERENCES}

Console, R., B. De Simoni and A. Di Sanza (1988): Riesame della relazione magnitudo-durata, in Proceedings of VII Yearly Meeting of the Gruppo Nazionale di Geo- 
fisica della Terra Solida (GNGTS), November 30-December 2, Rome, Italy, 51-62.

GASPERINI, P. (2002): Local magnitude revaluation for recent Italian earthquakes (1981-1996), J. Seismol., 6, 503-524.

GutenBerG-Richter, N. (1944): Frequency of earthquakes in California, Bull. Seismol. Soc. Am., 35, 185-188.

HabermanN, R.E. (1983): Telesesmic detection in the Aleutin Island Arc, J. Geophys. Res., 88, 5056-5064.

NutTLI, O.W. (1973): Seismic wave attenuation and magni- tude relations for Eastern North America, J. Geophys. Res., 78 (5), 876-885.

Richter, C.F. (1935): An instrumental earthquake magnitude scale, Bull. Seismol. Soc. Am., 25, 1-31

STEPP, J.C. (1972): Analysis of completenessof the earthquake sample in the Puget Sound area and its effect on statistical estimates of earthquake hazard, in Proceedings of the First Microzonation Conference, Seattle, U.S.A., 897-909. 